\title{
Nuclear Exclusion of p33ING1b Tumor Suppressor Protein: Explored in HCC Cells Using a New Highly Specific Antibody
}

\author{
Berna Sayan, Neset Cevdet Tolga Emre, Meliha Burcu Irmak, \\ Mehmet Ozturk, and Rengul Cetin-Atalay
}

\begin{abstract}
Mouse monoclonal antibodies (MAb) were generated against p33ING1b tumor suppressor protein. 15B9 MAb was highly specific in recognizing a single protein band of $\sim 33 \mathrm{kDa}$ endogenous p33ING1b protein from HCC cell lines and normal liver tissue by Western blot analysis and by immunoprecipitation. Although p33ING1b mutations are rarely observed in cancer, differential subcellular distribution and nuclear exclusion of p33ING1b were reported in different cancer types. Therefore we analyzed the expression and subcellular localization of p33ING1b in HCC cell lines using 15B9 MAb. So far, p33ING1b mutations or differential subcellular localization are not reported in HCC. In this study, by indirect immunofluorescence using MAb 15B9, we demonstrate that nuclear localization of p33ING1b was highly correlated with well-differentiated HCC cell lines whereas poorly differentiated HCC cells have nuclear exclusion of the protein. Moreover no association was observed between differential subcellular localization of p33ING1b and p53 mutation status of HCC cell lines. Hence our newly produced MAb 15B9 can be used for studying cellular activities of p33ING1b under normal and cancerous conditions.
\end{abstract}

\section{Introduction}

T HE ING1B PROTEIN IS A MEMBER of the tumor suppressor gene family involved in cell cycle, apoptosis, and senescence. ${ }^{(1,2)}$ The inhibitor of growth (ING) gene family comprises the ING1, ING2 ING3, ING4, and ING5 genes, which carry high homology in-between. ${ }^{(3)}$ Initially, the p33ING1b gene and then three other alternatively spliced variants (p47ING1a, p24ING1c, and p27ING1d) of ING1 gene were discovered. $^{(3,4)}$ It was shown that p33ING1b blocked cell cycle when ectopically over-expressed in various cell lines. Expression of the anti-sense construct of p33ING1b also resulted in enhanced transformation. ${ }^{(1)}$ Interaction of p33ING1b with PCNA upon UV irradiation in addition supports its involvement in apoptosis and DNA repair. Through its PHD zinc finger motif, the $\mathrm{p} 33 \mathrm{ING} 1 \mathrm{~b}$ protein was also suggested to be involved in chromatin remodeling and associated with histone acetyltransferase activities. ${ }^{(5)}$ The cellular activities of p33ING1b, along with its nuclear localization signal and PHD domain motif, strongly indicate that this protein acts in the nucleus. ${ }^{(6,7)}$ Moreover its interaction with $\mathrm{p} 53$ protein depends on nuclear localization of p33ING1b. Therefore, nuclear localization of p33ING1b is indispensable for its cellular function.

It is well known that tumor suppressor proteins show differential expression patterns or mutant products in cancer tissues. However p33ING1b mutations are rarely observed in human cancers. In breast, gastric esophageal blood, and brain tumors, p33ING1b was reported to be down-regulated. ${ }^{(8)}$ On the other hand, in melanoma, papillary thyroid carcinoma, and ductal breast carcinoma, increased protein levels in the cytoplasm was observed. ${ }^{(8)}$ These observations indicate that the involvement of p33ING1b in carcinogenesis cannot be explained by only gene mutations or altered expression. Subcellular targeting of proteins is crucial for their ultimate function for the cellular machinery. Therefore alterations in nuclear localization and eventual nuclear exclusion of p33ING1b protein were previously studied in correlation with carcinogenesis. ${ }^{(9-11)}$ In this study, we aimed to analyze subcellular distribution of the p33ING1b protein in HCC cell lines using our newly produced monoclonal antibodies $(\mathrm{MAb})$ against the p33ING1b protein. We demonstrated that p33ING1b protein was mislocalized in HCC cells, which are poorly differentiated.

\section{Materials and Methods}

\section{Expression and purification of GST-p33ING1b}

The oligonucleotide primers used for the PCR amplification of p33ING1b were 5'-AGACGTCGACAAATGTTGAGTCCT GCCAACG-3' (forward) and 5'-AGACAAGCTTCTACCTGT

Department of Molecular Biology and Genetics, Faculty of Science, Bilkent University, Ankara, Turkey. 
TGTAAGCCTCTC-3' (reverse). PCRs were performed using Pfu DNA Polymerase from Stratagene (La Jolla, CA) from MCF7 cell total cDNA. After cloning, the p33ING1b gene was verified by sequencing. Protein expression was induced by the addition of $0.1-0.2 \mathrm{mM}$ IPTG to actively growing DH5 $\alpha$ cells in LB medium at an A600 of 0.4-0.5. Incubation was continued for a further $3-4 \mathrm{~h}$ at $30^{\circ} \mathrm{C}$; then bacteria were harvested by centrifugation. Overexpressed GST-p33ING1b was purified by batch purification using Glutathione-Sepharose $4 \mathrm{~B}$ beads equilibrated in the sonication buffer $(50 \mathrm{mM}$ Tris$\mathrm{HCl}, 300 \mathrm{mM} \mathrm{NaCl}, 10 \%$ Glycerol (v/v), $7 \mathrm{mM}$ 2-ME [pH 7.8]). Elution was performed by incubating the bound resin with $5 \mathrm{mM}$ reduced glutathione in $50 \mathrm{mM}$ Tris- $\mathrm{HCl}$ (pH8.6), 200 $\mathrm{mM} \mathrm{KCl}$, and $7 \mathrm{mM}$ 2-ME for $10 \mathrm{~min}$ at room temperature.

\section{Immunization}

Purified $80 \mu \mathrm{g}$ GST-p33ING1b in emulsified Freund's complete adjuvant (Sigma Chemical, St. Louis, MO) was subcutaneously injected in 8-week-old female BALB/c mice. Two boosters of $80 \mu \mathrm{g}$ GST-p33ING1b in incomplete Freund's adjuvant at 3-week intervals were applied. Three days before fusion, intraperitoneal immunization with $60 \mu \mathrm{g}$ GSTp33ING1b was carried out.

\section{Cell fusion}

Hybridomas were produced by fusing splenocytes of an immunized BALB/c mouse with SP2 myeloma cells using PEG 5000 in 1:10 ratio. Cells were grown in RPMI-1640 (Biochrom AG, Berlin, Germany) supplemented with 20\% FBS $(\mathrm{v} / \mathrm{v}), 1 \mathrm{x}$ hypoxanthine-aminopterin-thymidine (HAT, Sigma). After 10-20 days, supernatants of growing hybridomas were screened with ELISA against purified GST-p33ING1b and GST proteins as negative control. Supernatants positive for GST-p33ING1b but not GST protein were selected then subcloned by limited serial dilution. Supernatants of the growing hybridomas were used for further studies.

\section{Tissue culture}

Different hepatoma cell lines were used to analyze the cellular properties of p33ING1b with respect to their differentiation status (well-differentiated cells: Huh7, HepG2, Hep3B, Hep3B-TR; poorly differentiated cells: Mahlavu, PLC/PRF/5, FOCUS, Sk-Hep1). ${ }^{(13)}$ Cells were grown in DMEM containing $10 \%$ fetal calf serum, $1 \%$ penicillin/streptomycin, and $1 \mathrm{x}$ nonessential amino acids.

\section{Western blot analysis}

Cells or frozen normal liver tissue were lysed by NP-40 lysis buffer (150 mM NaCl, 1.0\% NP-40, $50 \mathrm{mM}$ Tris [pH 8.0], protease inhibitor cocktail-Roche) and centrifuged at $13,000 \mathrm{rpm}$ at $4{ }^{\circ} \mathrm{C}$ for $30 \mathrm{~min}$. Thirty $\mu \mathrm{g}$ protein from each cell lysate was boiled for $5 \mathrm{~min}$ at $90^{\circ} \mathrm{C}$ with Laemmli buffer (62.5 mM Tris [pH 7.6], 2\% SDS, 10\% glycerol, BPB, 5\% 2- $\beta \mathrm{Me}$ ) and loaded on to $10 \%$ polyacrylamide gels. Transfer of the proteins to PVDF membrane (Millipore, Billerica, MA) was performed by Bio-Rad semi-dry transfer system (Bio-Rad, Hercules, CA). Membranes were blocked in TBS-T containing $3 \%$ milk powder. Commercial p33ING1b antibody (sc-7566goat polyclonal; Santa Cruz Biotechnology, Santa Cruz, CA) was diluted as recommended by the supplier. Mouse mono- clonal hybridoma supernatant was diluted 1:1 with TBS-T containing 3\% non-fat milk powder. HRP-conjugated antimouse (P0161, Dako, Glostrup, Denmark) and anti-goat (P0449, Dako) antibodies were used as the secondary antibodies. Amersham ECL-Plus kit (Amersham Pharmacia Biotech, Uppsala, Sweden) was used for the detection of the proteins.

\section{Immunoprecipitation}

Huh7 cells grown to $70 \%$ confluency were then starved in DMEM lacking methionine (Sigma) and labeled with $200 \mu \mathrm{Ci}$ [35S]Methionine (Amersham) in $4 \mathrm{~mL}$ medium for $2 \mathrm{~h}$. Cells were washed in ice-cold PBS, lysed in NP-40 lysis buffer, and centrifuged. $200 \mu \mathrm{g}$ protein from Huh7 cell lysate was immunoprecipitated with four different p33INGb monoclonal antibody producing hybridoma supernatants and protein G-sepharose.

\section{Immunofluorescence}

Different hepatoma cells $(200,000)$ were seeded on glass cover slips in 6-well dishes, then incubated overnight in Dulbecco's modified Eagle's medium with $10 \%$ fetal calf serum. Cells were fixed in $100 \%$ ice-cold acetone and saturated for $15 \mathrm{~min}$ in PBS-T (0.1\%) containing 3\% BSA. Anti-p33ING1 15B9 MAb supernatants were diluted at 1:1 in PBS-T and incubated for $1 \mathrm{~h}$. FITC-conjugated goat-anti-mouse (P0479, Dako) was used as the secondary antibody. Nuclear DNA was visualized by incubation with $3 \mu \mathrm{g} / \mathrm{mL}$ Hoechst 33258 (Sigma) for $5 \mathrm{~min}$ in the dark. Coverslips were then rinsed with distilled water, mounted on glass microscopic slides, and examined under fluorescent microscope (Zeiss, Göttingen, Germany).

\section{Results}

\section{Expression and purification of GST-p33ING1b fusion protein}

p33ING1b cDNA was PCR-amplified from the total cDNA obtained from the MCF7 breast adenocarcinoma cell line. This cell line was reported to show reduced expression of p33ING1b, but no mutations were observed. ${ }^{(1)}$ The sequence of $\mathrm{p} 33 \mathrm{ING} 1 \mathrm{~b} \mathrm{cDNA}$ was confirmed by sequencing. In order to produce p33ING1 protein, cDNA was subcloned to pGEX2TK-P expression vector. Expression and purification of GSTp33 fusion protein using Gluthatione-Sepharose 4B beads yielded a total protein amount of $\sim 2 \mathrm{mg}$ from $1 \mathrm{~L}$ culture media. Purified GST-p33ING1b was characterized by SDSPAGE (Fig. 1). After thrombin digestion, the p33ING1b protein was observed at the expected molecular weight (Fig. 1, lane 2).

\section{Production and characterization of different p33ING1b MAbs}

We generated four MAbs against p33ING1b protein from mice immunized with recombinant GST-p33ING1b protein by selective ELISA screening of antibody producing hybridomas using GST-p33ING1b. Four hybridoma clones (3G6, 9H9, 20H9, and 15B9) producing MAbs reactive against GSTfused p33ING1b were selected for further studies. Initially the MAbs were tested for their ability to recognize recombinant 


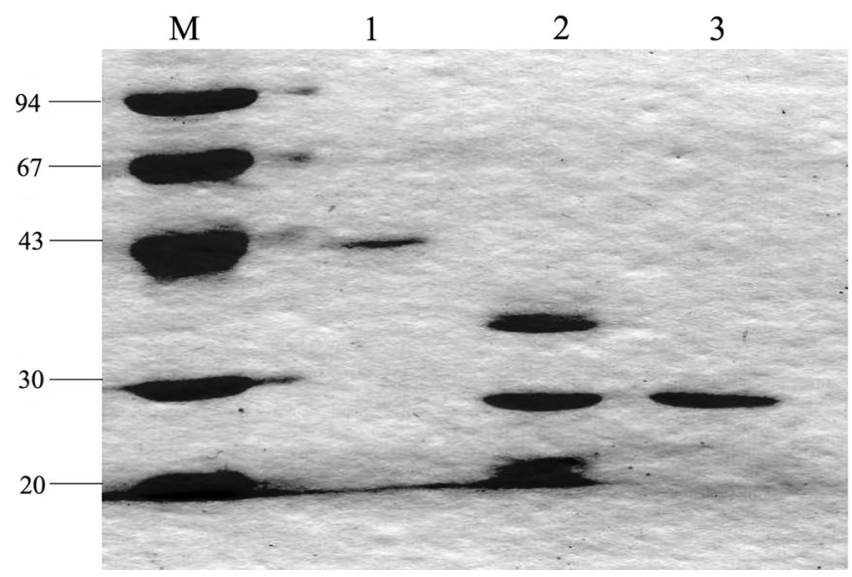

FIG. 1. SDS-PAGE analysis of GST-tagged p33ING. GSTp33ING1 fusion protein (lane 1), thrombin digested GSTp33ING1 fusion protein (lane 2), and purified GST protein was loaded to $12 \%$ SDS-PAGE and stained with CoomassieBriliant Blue. M, molecular weight marker.

GST-p33ING1b protein using Western immunoblotting technique (Fig. 2).

\section{Characterization of different p33ING1b MAbs}

Pure GST protein, GST-p33ING1b fusion protein, and cell lysate from two different hepatoma cell lines were used to compare the efficiency of four different hybridoma supernatants for the recognition of recombinant versus endogenous p33ING1b. For that purpose it was important to demonstrate that these hybridoma supernatants do not cross-react with the GST tag. Western blotting with 3G6, 9H9, 20H9, and 15B9 revealed that none of these MAbs from hybridoma supernatants cross-reacted with GST, but they all recognized the GST-p33ING1b fusion protein very efficiently (Fig. 2A). Surprisingly, only the 15B9 MAb was able to react with endogenous p33ING1b protein in two hepatoma cell lines (Fig. 2B), suggesting that the epitopes of the other three antibodies might be at the GST-p33ING1b fusion region. Furthermore when tested by immunoprecipitation, 15B9 was again able to recognize the endogenous p33ING1b protein (Fig. 2C). In addition, we checked the reactivity of 15B9 against mouse and rat tissues by Western blotting. Our results demonstrated that our newly formed, highly specific antibody raised against human-p33ING1 recognizes a single band of $33 \mathrm{kDa}$ mouse and rat p33ING1b protein (data not shown).

\section{Expression of p33ING1b in hepatoma cell lines}

Different hepatoma cells were grown in tissue culture. Proteins were extracted from each cell line and then Western blot was performed. All the cell lines, with some minor differences, expressed p33ING1 protein (Fig. 3A). Similar expression of p33ING1b in nine hepatoma cell lines and normal liver were also confirmed by RT-PCR (data not shown). In addition we analyzed the protein levels in Huh7, HepG2, Hep3B, and Mahlavu cells compared with calnexin housekeeping protein. We did not observe significant differential p33ING1b gene expression in these samples either (Fig. 3B). Considering cellular functions of p33ING1b and its interac-
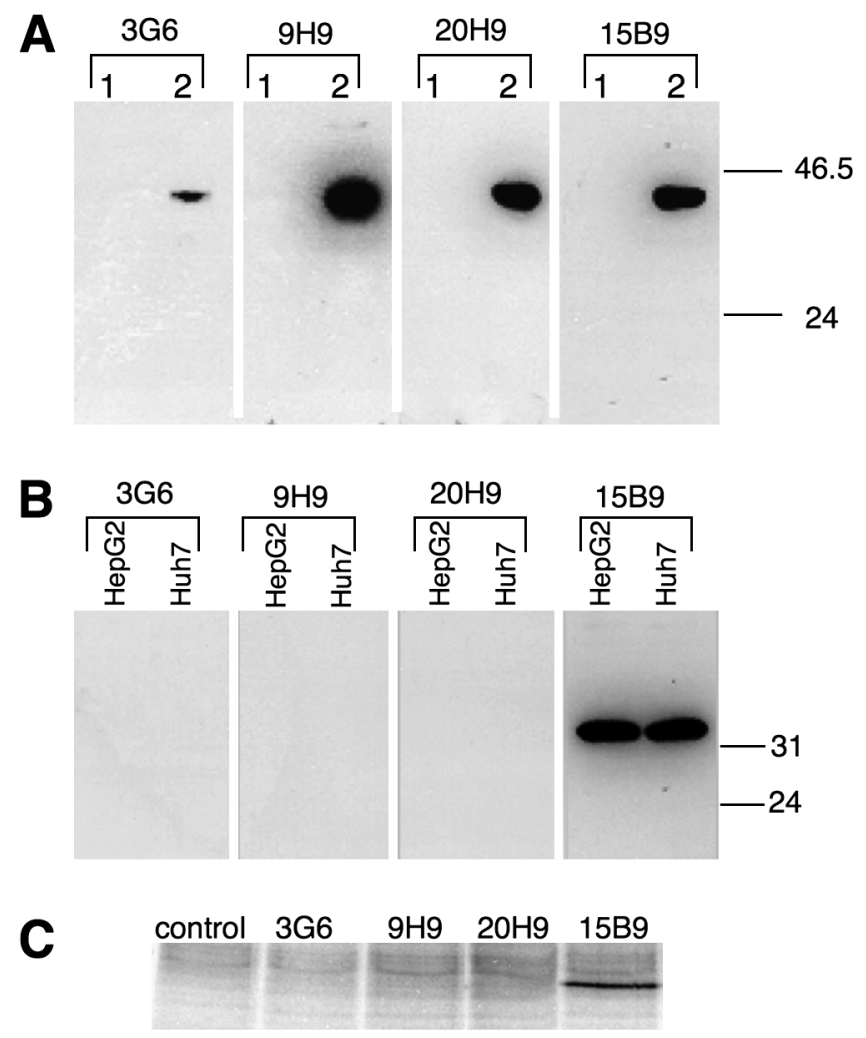

FIG. 2. Analysis of different hybridoma supernatants. (A) Western blotting with 3G6, 9H9, 20H9, and 15B9 hybridoma supernatants for the recognition of recombinant GST protein (1) versus GST-p33ING fusion protein (2). (B) Immunoprecipitation of endogenous p33ING1 protein from metabolically labeled Huh7 hepatoma cells. Protein extracted from these cells was immunoprecipitated by the four different hybridoma supernatants. Control represents the IP in which no primary antibody was used. (C) Western blot analysis of the hybridoma supernatants on endogenous p33ING1 protein from Huh7 and HepG2 cells.

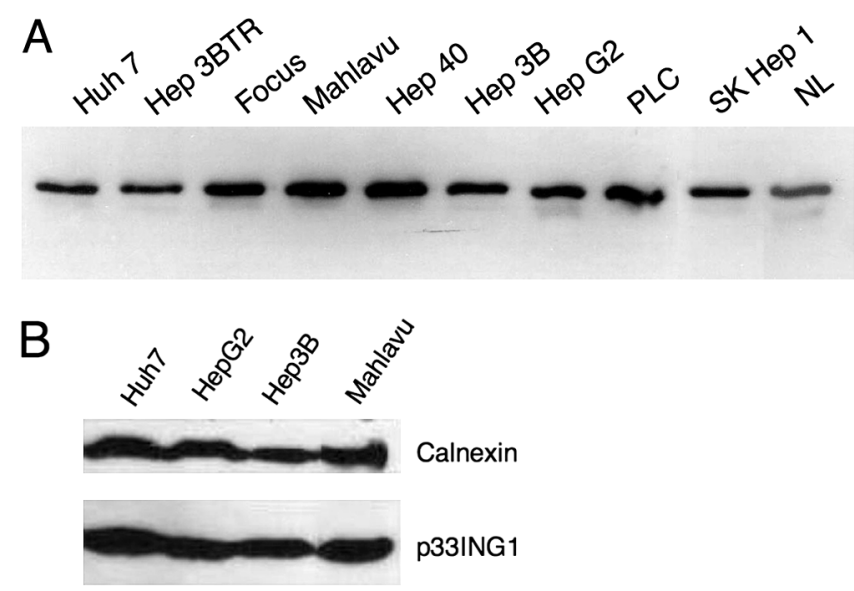

FIG. 3. Expression of p33ING1 in different hepatoma cell lines. (A) Thirty $\mu \mathrm{g}$ cell lysate or normal liver extract from frozen tissue (NL) was loaded to $12 \%$ SDS-PAGE and subjected to Western blot analysis with MAb 15B9. (B) Comparable p33ING1 protein expression in Huh7, HepG2, Hep3b, and Mahlavu cells. Blot was reprobed with anti-calnexin antibodies to confirm equal protein loading in each lane. 
FIG. 4. Analysis of subcellular distribution of p33ING1 protein detected by immunofluorescence analysis using MAb 15B9 on a selected set of cells carrying wild type (SKHep1 and HepG2), codon 220 mutant (Huh7), codon 249 mutant (Mahlavu and PLC), and cells without p53 (Hep3B, Hep3B-TR, and FOCUS).

Nuclear exclusion of p33ING1 protein can be observed by comparison with nuclear DNA that was visualized by Hoechst 33258 counterstaining. Color images available online at www .liebertonline.com/hyb.
HepG2
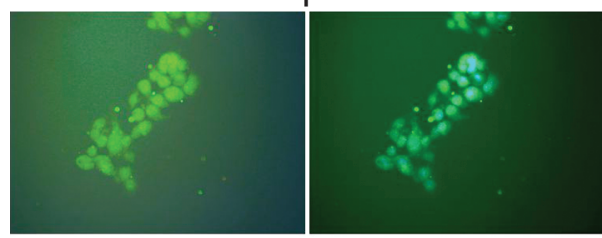

Hep3B

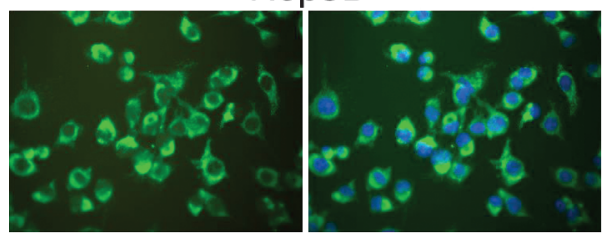

SKHep1
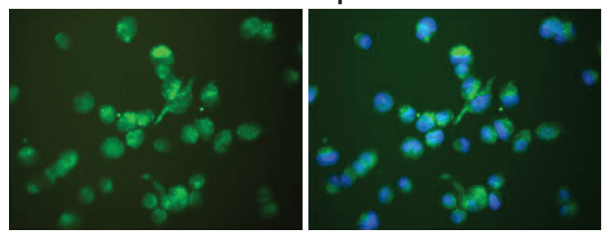

PLC/PRF/5

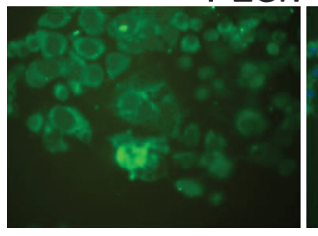

Huh7

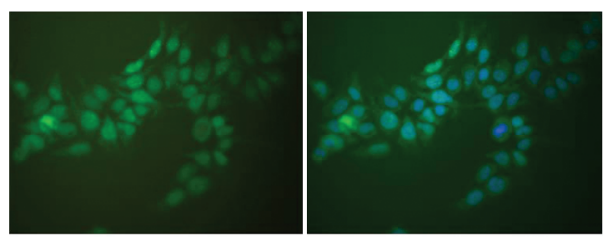

Hep3B-TR

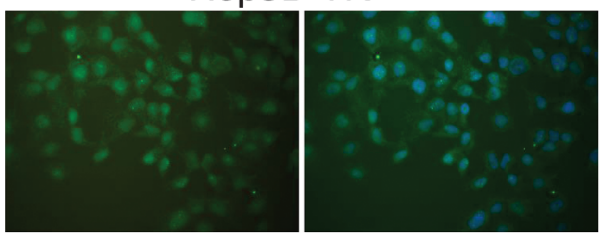

Mahlavu

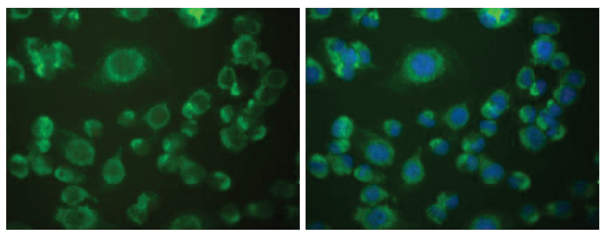

FOCUS
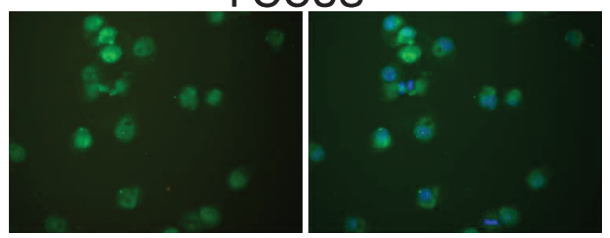

tion with nuclear proteins involved in histone modification, we then analyzed the subcellular distribution of ING1b on a set of hepatoma cells with 15B9 antibody by indirect immunofluorescence.

\section{Subcellular distribution of p33ING1b in HCC cell lines}

Well-differentiated hepatoma cells-Huh7, HepG2, Hep3B, Hep3B-TR - and the poorly differentiated cellsMahlavu, PLC/PRF/5, FOCUS, Sk-Hep1-were compared in their p33ING1b subcellular distribution. Huh7, HepG2, Hep3B-TR cells had nuclear p33ING1b expression, whereas Hep3B, Mahlavu, PLC/PRF/5, FOCUS, Sk-Hep1 revealed strong nuclear exclusion of the protein (Fig. 4). The strong extranuclear staining may indicate cytoplasmic accumulation of the p33ING1b protein. However we did not observe a correlation between nuclear localization of p33ING1b and p53 mutations status of these cell lines (Table 1).

\section{Discussion}

In this study we generated four MAbs against GSTp33ING1b protein. MAb 15B9 was the only antibody that recognized native $\mathrm{p} 33 \mathrm{ING} 1 \mathrm{~b}$ protein. Western blot analysis of HCC cell lines Huh7 and HepG2 (Fig. 2) and of mouse and rat tissues (data not shown) yielded a single protein band of $\sim 33 \mathrm{kDa}$. Our data indicates that MAb 15B9 is highly specific to p33INGb isoform since we observed only a single

Table 1. Subcellular Dstribution of p33ing1 in Relation to p53 Status of Hepatoma Cells

\begin{tabular}{|c|c|c|c|}
\hline Cell line & Differentiation status & $\begin{array}{l}\text { p33 nuclear } \\
\text { localization }\end{array}$ & p53 status \\
\hline HepG2 & Well differentiated & Nuclear & Wild type \\
\hline Huh7 & Well differentiated & Nuclear & Mutant (Tyr220Cys) \\
\hline Hep3B-TR & Well differentiated & Nuclear & Null \\
\hline Hep3B & Well differentiated & Excluded & Null \\
\hline Sk-Hep1 & Poorly differentiated & Excluded & Wild type \\
\hline Mahlavu & Poorly differentiated & Excluded & Mutant (Arg249Ser) \\
\hline $\mathrm{PLC} / \mathrm{PRF} / 5$ & Poorly differentiated & Excluded & Mutant (Arg249Ser) \\
\hline FOCUS & Poorly differentiated & Excluded & Null \\
\hline
\end{tabular}


protein band from Western blot analysis of Huh7 and HepG2 cells (Fig. 3C). The epitope of the MAb $15 B 9$ must lie on the Nterminus of the p33ING1b, where there is the least homology between the p33ING1 isoforms. ${ }^{(3)}$ Therefore MAb 15B9 can be used as a highly specific antibody for p33ING1b.

Studies on p33ING1 protein showed infrequent mutation or $\mathrm{LOH}$ although it was reported as a putative tumor suppressor. ${ }^{(1)}$ In general tumor suppressor proteins show either differential expression patterns or an inactive mutant product in various types of cancers. p33ING1 down-regulation was previously reported in breast, gastric, esophageal, blood, and brain cancers, whereas in melanoma, papillary thyroid carcinoma and ductal breast carcinoma increased cytoplasmic protein levels. ${ }^{(8)}$ In addition, p33ING1 was reported to be mainly localized to the nucleus in most of the normal tissues with the exception of hepatocytes but not bile duct epithelial cells in formalin-fixed sections. ${ }^{(14)}$ Therefore, in this study using our newly generated highly specific p33ING1b MAb 15B9, we analyzed p33ING1b differential expression and eventually its subcellular distribution in HCC cell lines in correlation with their p53 status. We did not observe significant differential expression in hepatoma cell lines (Fig. $3 \mathrm{~A}$ and $\mathrm{B}$ ). Normal liver tissue from a frozen sample had similar p33ING1 expression. Previously using different MAbs, low or no p33ING1 expression was reported with formalin-fixed liver tissue samples by immunostaining. ${ }^{(14,15)}$ In our study we did not observe a significant difference in p33ING1 expression in frozen normal liver tissue when compared to hepatoma cell lines with Western blot analysis (Fig. 3A). The comparable expression that we observed in normal liver tissue may be due to either the use of 15B9 MAb, which is highly reactive to p33ING1b, or the use of freshly frozen liver tissue for protein extraction. In addition the comparable expression of p33ING1b in 15 hepatoma cell lines and normal liver was also confirmed by RT-PCR. Considering previous reports about the p33ING1b as putative tumor suppressor and our results indicating that the involvement of p33ING1b in cancer cannot be explained by only gene mutations or altered expression, subcellular distribution through nuclear exclusion of $\mathrm{p} 33 \mathrm{ING} 1 \mathrm{~b}$ protein may play an important role in its cellular activities. Therefore we further analyzed subcellular distribution of p33ING1b in eight HCC cell lines. Well-differentiated hepatoma Huh7, HepG2, Hep3BTR cells had nuclear p33ING1b and in poorly differentiated Mahlavu, PLC/PRF/5, FOCUS, Sk-Hep1 cells, p33ING1b was sequestered in the cytoplasm of the nucleus. Differential distribution of p33ING1b was independent of p53 status of these cell lines (Table 1). Our data on the p53 independent differential subcellular distribution of p33ING1b are in correlation with previous studies, which also reported p53 independent activities of p33ING1b in cell proliferation inhibition. ${ }^{(16,17)}$ ING family proteins have been reported to be involved in chromatin modeling through their interactions with histone modifying protein complexes. ${ }^{(19-21)}$ Recent studies on the histone modification demonstrated the importance of chromatin remodeling in gene expression in cancer cells. ${ }^{(22)}$ Involvement of p33ING1b in carcinogenesis so far was not fully explained by mutations or differential expression studies. However, recently, differential subcellular distribution and exclusion of p33ING1b were reported in melanoma, acute lymphoblastic leukemia, invasive breast cancer, and oral squamous cell carcinomas. ${ }^{(9-12)}$ In this study we report that poorly differentiated HCC cell lines have nuclear exclusion of p33ING1. In conclusion these findings indicate that expulsion of p33ING1b from the nucleus in invasive carcinoma may explain the molecular involvement of $\mathrm{p} 33 \mathrm{ING} 1 \mathrm{~b}$ in carcinogenesis in support of its previously described function as tumor suppressor.

\section{Acknowledgment}

We thank Berra Erkosar for laboratory assistance. This work was supported by the Scientific and Technical Research Council of Turkey (TÜBITAK).

\section{References}

1. Garkavtsev I, Kazarov A, Gudkov A, and Riabowol K: Suppression of the novel growth inhibitor p33ING1 promotes neoplastic transformation. Nat Genet 1996;14:415-420.

2. Soliman MA, and Riabowol K: After a decade of study-ING, a PHD for a versatile family of proteins. Trends Biochem Sci 2007;32:509-519.

3. He GH, Helbing CC, Wagner MJ, Sensen CW, and Riabowol K: Phylogenetic analysis of the ING family of PHD finger proteins. Mol Biol Evol 2005;22:104-116.

4. Gunduz M, Ouchida M, Fukushima K, Hanafusa H, Etani T, Nishioka S, Nishizaki K, and Shimizu K: Genomic structure of the human ING1 gene and tumor-specific mutations detected in head and neck squamous cell carcinomas. Cancer Res 2000;60:3143-3146.

5. Vieyra D, Loewith R, Scott M, Bonnefin P, Boisvert FM, Cheema P, Pastyryeva S, Meijer M, Johnston RN, BazettJones DP, McMahon S, Cole MD, Young D, and Riabowol K: Human ING1 proteins differentially regulate histone acetylation. J Biol Chem 2002;277:29832-29839.

6. Nouman GS, Anderson JJ, Lunec J, and Angus B: The role of the tumour suppressor p33 ING1b in human neoplasia. J Clin Pathol 2000;56:491-496.

7. Gunduz M, Gunduz E, Rivera RS, and Nagatsuka H: The inhibitor of growth (ING) gene family: potential role in cancer therapy. Curr Cancer Drug Targets 2008;8:275-284.

8. Gong W, Suzuki K, Russell M, and Riabowol K: Function of the ING family of PHD proteins in cancer. Int J Biochem Cell Biol 2005;37:1054-1065.

9. Nouman GS, Anderson JJ, Mathers ME, Leonard N, Crosier $\mathrm{S}$, Lunec J, and Angus B: Nuclear to cytoplasmic compartment shift of the p33ING1b tumour suppressor protein is associated with malignancy in melanocytic lesions. Histopathology 2002;40:360-366.

10. Nouman GS, Anderson JJ, Wood KM, Lunec J, Hall AG, Reid MM, and Angus B: Loss of nuclear expression of the p33(ING1b) inhibitor of growth protein in childhood acute lymphoblastic leukaemia. J Clin Pathol 2002;55:596601.

11. Nouman GS, Anderson JJ, Crosier S, Shrimankar J, Lunec $\mathrm{J}$, and Angus B: Downregulation of nuclear expression of the p33(ING1b) inhibitor of growth protein in invasive carcinoma of the breast. J Clin Pathol 2003;56:507-511.

12. Zhang JT, Wang DW, Li QX, Zhu ZL, Wang MW, Cui DS, Yang YH, Gu YX, and Sun XF: Nuclear to cytoplasmic shift of p33(ING1b) protein from normal oral mucosa to oral squamous cell carcinoma in relation to clinicopathological variables. J Cancer Res Clin Oncol 2008;134:421-426.

13. Sayan AE, Sayan BS, Findikli N, and Ozturk M: Acquired expression of transcriptionally active p73 in hepatocellular carcinoma cells. Oncogene 2001;20:5111-5117. 
14. Nouman GS, Angus B, Lunec J, Crosier S, Lodge A, and Anderson JJ: Comparative assessment expression of the inhibitor of growth 1 gene (ING1) in normal and neoplastic tissues. Hybrid Hybridomics 2002;21(1):1-10.

15. Zhu Z, Lin J, Qu JH, Feitelson MA, Ni CR, Li FM, and Zhu $\mathrm{MH}$ : Inhibitory effect of tumor suppressor p33(ING1b) and its synergy with p53 gene in hepatocellular carcinoma. World J Gastroenterol 2005;11(13):1903-1909.

16. Gunduz M, Gunduz E, Rivera RS, and Nagatsuka H: The inhibitor of growth (ING) gene family: potential role in cancer therapy. Curr Cancer Drug Targets 2008;8:275-284.

17. Cheung KJ Jr, Bush JA, Jia W, and Li G: Expression of the novel tumour suppressor p33(ING1)is independent of p53. Br J Cancer 2000;83:1468-1472.

18. Tsang FC, Po LS, Leung KM, Lau A, Siu WY, and Poon RY: ING1b decreases cell proliferation through p53-dependent and independent mechanisms. FEBS Lett 2003;553:277-285.

19. Skowyra D, Zeremski M, Neznanov N, Li M, Choi Y, Uesugi M, Hauser CA, Gu W, Gudkov AV, and Qin J: Differential association of products of alternative transcripts of the candidate tumor suppressor ING1 with the mSin3/HDAC1 transcriptional corepressor complex. J Biol Chem 2001;276:8734-8739.
20. Feng X, Hara Y, and Riabowol K: Different HATS of the ING1 gene family. Trends Cell Biol 2002;12:532-538.

21. Doyon Y, Cayrou C, Ullah M, Landry AJ, Côté V, Selleck W, Lane WS, Tan S, Yang XJ, and Côté J: ING tumor suppressor proteins are critical regulators of chromatin acetylation required for genome expression and perpetuation. Mol Cell 2006;21:51-64.

22. Esteller M: Cancer epigenomics: DNA methylomes and histone-modification maps. Nat Rev Genet 2007;8:286-298.

Address reprint requests to: Rengul Cetin-Atalay, M.D., Ph.D. Department of Molecular Biology and Genetics Faculty of Science Bilkent University 06533 Ankara Turkey

Email: rengul@bilkent.edu.tr

Received: June 30, 2008 Accepted: August 21, 2008 